\title{
Indirect Measurement of Shielding Effectiveness of an Enclosure for a Security Camera
}

\author{
Petr Skočík ${ }^{1}$, Martin Pospíšilík ${ }^{1}$, Vojtěch Křesálek ${ }^{1}$, Milan Adámek ${ }^{2}$ \\ ${ }^{1}$ Department of Electronics and Measurement, Faculty of Applied Informatics, Tomas Bata University in Zlin, \\ Nad Stráněmi 4511, 76005 Zlín, Czech Republic, skocik@utb.cz \\ ${ }^{2}$ Department of Security Engineering, Faculty of Applied Informatics, Tomas Bata University in Zlin, \\ Nad Stráněmi 4511, 76005 Zlín, Czech Republic
}

\begin{abstract}
This paper presents the results of our experiment, which consisted in measurement of the shielding effectiveness of a camera enclosure that was designed and constructed in order to increase the susceptibility of a camera against external electromagnetic fields. All activities, from defining of requirements to final design and measurements, were performed in the Laboratory of Electromagnetic Compatibility at the Faculty of Applied Informatics of Tomas Bata University in Zlín. The hereby described approach allowed to utilize standardized laboratory equipment that is normally used for device susceptibility test to radiated electric field. The measurement was made inside a semi-anechoic room additionally damped by absorbers placed on its floor. When configuring the experiment, relevant EMC standards EN 55016 and EN 61000-4-3 were considered. Although the uncertainty tolerances, allowed by the relevant standards, were quite high for measurements in such rooms, the results obtained by the experiment matched the theoretical expectations quite satisfactorily.
\end{abstract}

Keywords: Electric field measurement, security camera, shielding effectiveness, indirect measurement,

\section{INTRODUCTION}

Security surveillance cameras are a widely used tool for monitoring of various spaces. Although all the security cameras must be constructed in accordance with relevant standards including electromagnetic compatibility, their level of electromagnetic susceptibility to external electromagnetic fields may not be sufficient in all possible cases of their operation. In terms of electromagnetic susceptibility, the relevant standards, such as EN 62676 or EN 50130, contain references to generic EMC standards as EN 61000-6-1 (electromagnetic susceptibility) or EN 61000-6-3 (electromagnetic interference). Nevertheless, in specific cases it may be appropriate to increase the camera's immunity beyond the requirements of the standards.

The increase of the camera's immunity can be realized through an additional shielding cover. However, this approach is not entirely trivial, as the electromagnetic waves are to be suppressed but the passage of light that is necessary to take the picture must be ensured. Moreover, the camera inserted inside the shielding cover represents an inhomogeneity inside the shielded space that has a great impact on the shielding effectiveness. In this paper a description of the method of replacing the camera inside the shielding cover by an isotropic field probe of similar dimensions is described, as well as the results obtained by measurements in the laboratory.

The theory of an electromagnetic shielding [1] is an extremely large scientific field. Although the theory of electromagnetic field on a macroscopic scale [2] has been known well for a long time, in practice, it is not trivial to find an exact solution of the Maxwell's equations for specific technical applications. Moreover, when considering the properties of the electromagnetic shielding, not only the theory of electromagnetic field but also the knowledge of a relevant materials science must be considered. Therefore, different approximation approaches have been developed in accordance with the specific requirements of various technical applications. For example, in paper [3] the effects of material type and fabrics layers on the shielding properties of textile materials are discussed. The shielding effectiveness of a laminate structure in combination with different materials is discussed in paper [4]. The properties of polymer materials in relation to the electromagnetic shielding are described in paper [5].

Because this paper describes an experiment that deals with the measurement of shielding effectiveness of a metal cabinet that was constructed in order to improve the electromagnetic susceptibility of a security camera [6], let us focus our attention on examining the shielding properties of metal 
enclosures with necessary technological cable penetrations. A comprehensive discussion on this topic may be found in [7]. An example of a description of measurement of shielding effectiveness of materials according to the standards MILSTD 285 and IEEE STD 299-2006 can be found in [8].

It is worth mentioning that the possibility to blind a security camera by strong electromagnetic field represents a security risk, which is currently not receiving adequate attention [9] although this possibility is real and the experiment on this topic is easy to be performed in any EMC laboratory [10].

\section{SUBJECT \& METHODS}

According to IEEE 299 standard [11], the shielding effectiveness is defined as: "The ratio of the signal received (from a transmitter) without the shield, to the signal received inside the shield; the insertion loss when the shield is placed between the transmitting antenna and the receiving antenna." Considering the levels are measured as a voltage at the antenna terminals, the shielding effectiveness $S E$ can be expressed as follows:

$$
S E=20 \log \frac{\left|V_{o}\right|}{\left|V_{S}\right|}
$$

Where $V_{o}$ is the magnitude of the voltage measured at the terminals of an antenna with no enclosure present and $V_{s}$ is the magnitude of the voltage at the terminals of the same antenna when the antenna is placed inside the shielding enclosure. Assuming the voltages at the terminals of the measuring antenna are proportional to the intensities of the electric field, the following equation for shielding effectiveness can also be considered:

$$
S E=20 \log \frac{\left|E_{o}\right|}{\left|E_{S}\right|}
$$

Where $E_{o}$ is the intensity of the electric field when no shielding enclosure is applied while $E_{s}$ is the intensity of the electric field inside the shielding enclosure. If the power of the electric field is considered, the following alternative to (1) and (2) can be used:

$$
S E=10 \log \left(\frac{\left|E_{o}\right|}{\left|E_{S}\right|}\right)^{2}=10 \log \frac{P_{o}}{P_{S}}
$$

\section{A. Theory of shielding mechanism}

The complete theory of electromagnetic shielding was derived from Maxwell's equations by S. A. Schelkunoff [12]. Different interpretations of this theory have emerged over time. According to [13], the simplified description of the shielding effect of a metal plane can be found in Fig.1.

When the incident wave approaches the surface of the shielding material, part of the energy is reflected back to the space in front of the material and part of the energy pervades further. In fact, the shielding plane creates two interfaces. One between the free space and the shielding material and the second one between the shielding material and the inner space of the shielding enclosure.

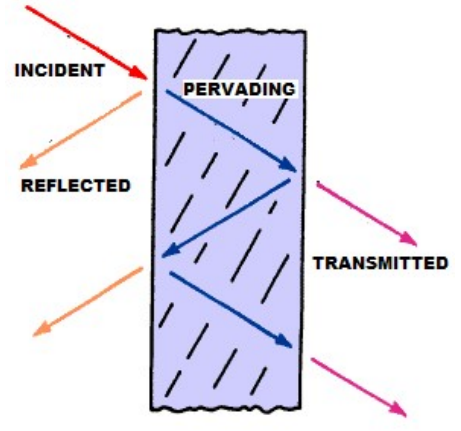

Fig.1. Principle of attenuation caused by means of multiple reflections. Incident, reflected, pervading, and transmitted waves are shown [13].

Therefore, the energy of the pervading wave is split again at the second interface and only a part of it reaches the inner space of the shielding enclosure. In Fig.1., this part is marked as "transmitted", while the second part is a reflection between the interfaces. The reflections inside the material can further be partially transmitted to both sides of the material. Mathematically, this mechanism can be described as follows:

$$
S E_{d B}=R_{d B}+A_{d B}+M_{d B}
$$

Where $R$ is the attenuation of the electromagnetic wave on the interface of different impedances, $A$ is the attenuation caused by absorption of the material (heat loss) and $M$ is the attenuation caused by multiplicated reflections.

Considering the space in front of the shielding material (left side in Fig.1.) is denoted as $\alpha$, the space behind the shielding material (right side in Fig.1.) is denoted as $\beta$ and the material is denoted as $M$, according to [13] the attenuation can be expressed by means of impedances of the pertinent environments as follows:

$$
R=20 \log \left|\frac{Z_{\alpha}+Z_{M}}{2 \cdot Z_{M}} \cdot \frac{Z_{M}+Z_{\beta}}{2 \cdot Z_{\beta}}\right|[d B]
$$

The absorption of the energy (heat loss) is determined by the intrusion depth $\delta$.

$$
\begin{gathered}
A=20 \log \left|e^{\frac{t}{\delta}}\right|[d B] \\
\delta=\sqrt{\frac{2}{\omega \mu \sigma}}
\end{gathered}
$$

In (6) and (7), $t$ is the thickness of the material [m], $\delta$ is the intrusion depth $[\mathrm{m}], \sigma$ is the material conductivity $\left[{\left.\mathrm{S} . \mathrm{m}^{-1}\right], \mu \text { is }}^{-1}\right.$ the material permeability $\left[\mathrm{H}_{\mathrm{m}} \mathrm{m}^{-1}\right]$, and $\omega$ is the wave frequency [rad. $\mathrm{s}^{-1}$ ].

Finally, let us consider the attenuation caused by multiplied reflections inside the material, when the wave enters the material and is reflected back inside the material and then is finally reflected again and passes through the interface to the inner part of the enclosure. If the impedance of the environment on both sides of the shielding material is $Z_{0}=Z_{\alpha}=Z_{\beta}$, the attenuation $M$ can be expressed as follows: 


$$
M=20 \log \left|1-\left(\frac{Z_{O}+Z_{M}}{Z_{O}+Z_{M}}\right)^{2} \cdot e^{-\frac{2 t}{\delta}} \cdot e^{-j \frac{2 t}{\delta}}\right|[d B]
$$

More information on the theory of shielding mechanism may be found in [1], [8], [13].

\section{B. Practical issues}

Practical issues on construction of the shielding enclosure are described in [6] in details. Once a shielding enclosure is constructed, problems arising from practical implementation will arise. In practice, the shielding effectiveness is decreased by imperfections in joints of the parts of the cabinet, by every penetration, etc. Moreover, when the connection of the device laced inside the shielding enclosure is created by means of metallic cables, the electromagnetic interference is dragged inside.

Moreover, measurement of the field intensity inside the enclosure is non-trivial, as any antenna or sensor placed inside the enclosure affects the displacement of the field significantly. This issue is exhaustively discussed in [7]. Once the EM wave enters the inner space of the enclosure, reflections leading to the occurrence of standing waves occur. Although the amount of energy released inside may be low, the occurrence of standing waves may, in some points in the space of the enclosure, decrease its shielding effectiveness significantly. For large systems, these effects are described in [14] and [15].

Measurement of the field properties inside the shielding chamber is a typical discipline in which the measurement itself affects the result considerably [16]. Generally, the issues on reverberant behaviour of the inner space of the shielding enclosures are described in [17] and [18]. Moreover, the polarization of the measuring antenna and the illuminating field must be considered [11].

Let us briefly describe the conclusions that may be found in the above-mentioned literature:

1. The displacement of the EM energy inside the shielding enclosure is generally irregular.

2. The measuring antenna placed in the enclosure affects the field displacement quite considerably.

\section{Shielding enclosure for a camera}

Other specific issues arise when the shielding enclosure is constructed for a camera. At least part of the enclosure must be transparent to light. Great effort has been devoted to finding materials suitable for this purpose and the results are exhaustively described in [6].

A construction of the shielding enclosure is depicted in Fig.2. It is obvious that the front side of the enclosure is made of a transparent material. According to the experiments described below, a technical fabric made of metal was used for this purpose. The rest of the construction is made of a steel plate.

The reasons for using this fabric and its detailed specifications are to be found in [6]. A compromise between the shielding effectiveness of the material and the transparency for the image captured by the camera was chosen.

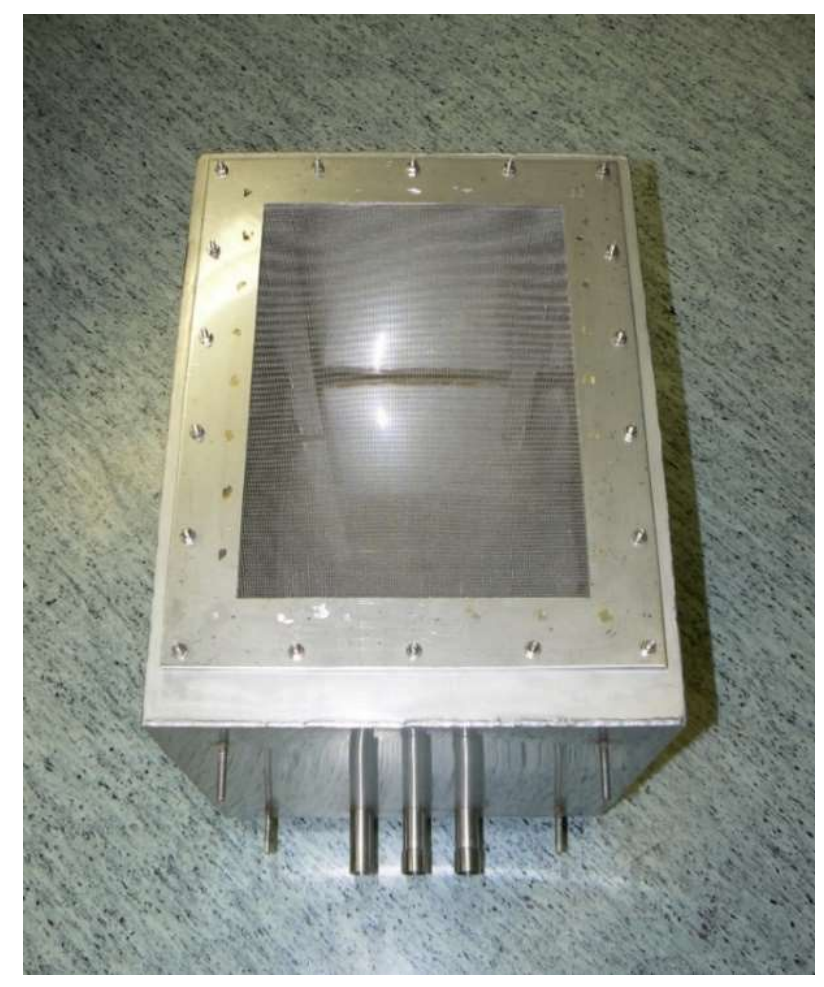

Fig.2. Shielding enclosure for a security camera.

\section{Expected shielding effectiveness of the materials}

During the design and construction process, the shielding effectiveness of construction materials was continuously verified by a measuring method using coaxial lines. This method consists in insertion of the measured material in a space between two flanged coaxial lines. The transmission through the coaxial lines is then measured with and without the material and afterwards, the shielding effectiveness of the material is calculated according to the obtained results. This method is described for example in [19] in a very detailed way. Technical standards for this measurement method are defined in [20]. The test setup used in our laboratory is depicted in Fig.3.

The results obtained by the method using coaxial transmission line are depicted in Fig.4. The following materials were tested:

1. Metal plate used for the construction of the enclosure.

2. Technical fibre TT-0,400-0,22-1.4301-1000.

3. Technical fibre TT-0,630-0,25-1.4401-1000.

4. Technical fibre TT-1,250-0,40-1.4301-1000.

Within the frequency range from $250 \mathrm{MHz}$ up to $3 \mathrm{GHz}$, the expected levels of shielding effectiveness were $S E_{M P} \approx 68.9 \mathrm{~dB}$ for the solid metal plate and $S E_{T F} \approx 46.5 \mathrm{~dB}$ for various technical fibres. Because the performance of all of the technical fibres was approximately similar, the type TT 0,630-0,25-1.4401-1000 was selected for the construction of the cabinet, as practical tests showed that it was sufficiently transparent in order not to affect the image quality of the camera [6]. 
According to the results of the measurement depicted in Fig.4., the following assumptions were made: considering the material with the worst performance, the shielding effectiveness of the whole enclosure for the security camera $S E_{\text {total }}$ was expected to be approximately $55 \mathrm{~dB}$, i.e. some value between $S E_{M P}$ and $S E_{T F}$.

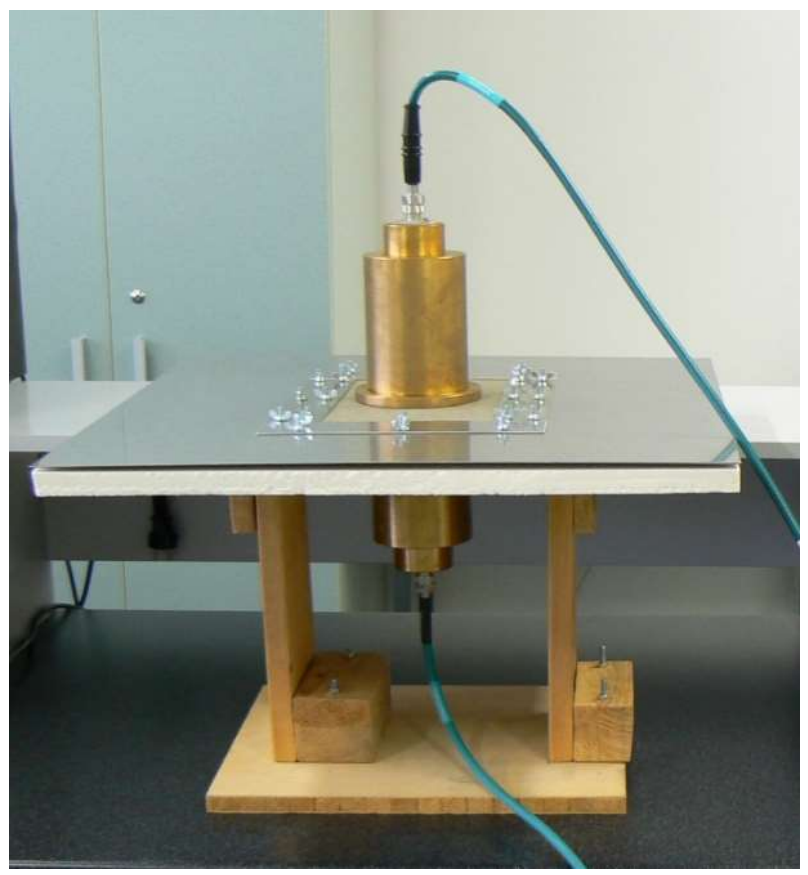

Fig.3. Test setup for measurement of shielding effectiveness of metal fabrics.

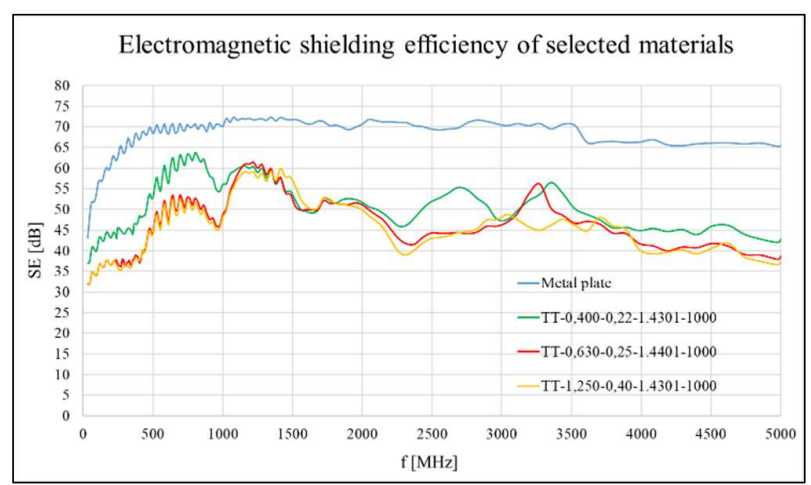

Fig.4. Electromagnetic shielding effectiveness of selected materials.

\section{EXPERIMENTAL SETUP AND DESCRIPTION}

This chapter describes an experiment that was conducted in the Laboratory of Electromagnetic Compatibility of the Faculty of Applied Informatics at Tomas Bata University in Zlín. The aim of the experiment was to verify the expected shielding effectiveness of the shielding enclosure for a security camera, described in the chapter above. The expected level of the shielding effectiveness was as high as $55 \mathrm{~dB}$.

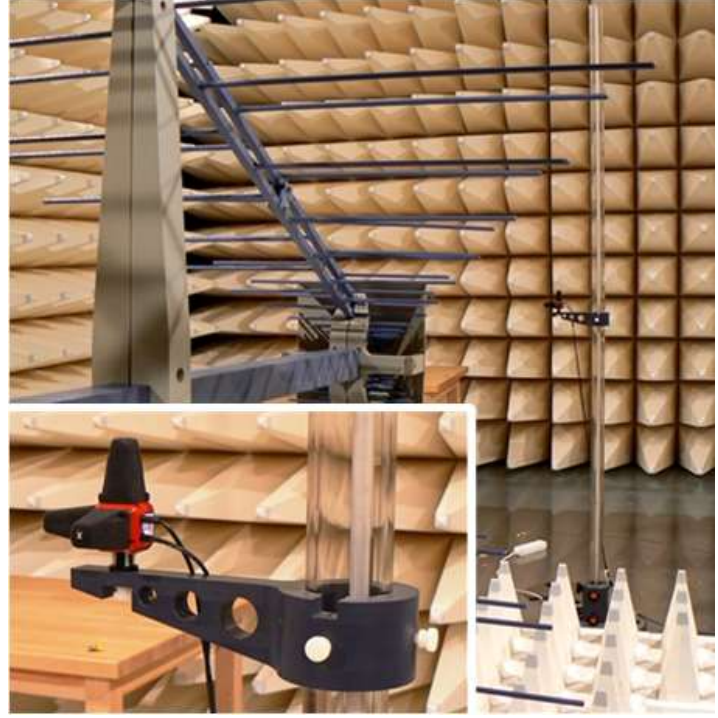

Fig.5. Experimental setup without the enclosure.

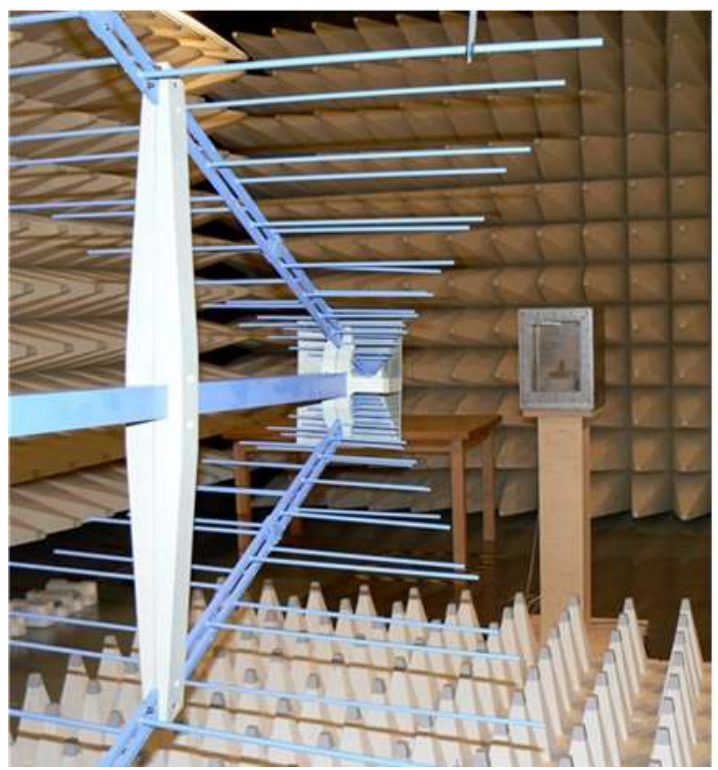

Fig.6. Experimental setup with the enclosure.

Due to the dimensions of the enclosure and its cable penetrations, in the laboratory, there was no other possible choice than the three-dimensional anisotropic E-field probe ETS Lindgren HI-6105 [21]. It is small enough to be inserted inside the enclosure and its connection is realized by means of optical cables which reduces the risk of dragging interferences to the inner space. Moreover, this field probe gives information on field intensity according to three axes in space $(x, y, z)$ that is afterwards processed by the controlling system. As the displacement of the electric field inside the enclosure depends on the parameters of illuminating wave and the reflections inside the enclosure [7], utilization of an omnidirectional field probe eliminates the need for changing the polarity and direction of the measuring antenna inside the 
enclosure. Compared to the dimensions of the enclosure, the dimensions of the probe are quite small. Therefore, lower influence on the internal field displacement was expected, compared to the utilization of a conventional antenna. On the other hand, the E-field probe shows considerable disadvantage consisting in very low sensitivity. The real noise level of its output is approximately $10 \mathrm{mV} / \mathrm{m}$.

The experiment was located in a semi-anechoic room Frankonia SAC 3+. This room is primarily designed for measurement of EMC interferences according to the standards based on the generic standard EN 61000-6-3 and for testing of electromagnetic susceptibility of electronic devices according to the standard EN 61000-4-3. In order to decrease the level of reflections inside the semi-anechoic room, its floor was covered by pyramidal absorbers [14]. As a source of the electromagnetic field, the Rohde \& Schwarz HL 046E transmitting antenna was used. The antenna was placed in a distance of 3 meters from the centre of the measured enclosure. The enclosure was mounted on a stand that elevated the centre of the enclosure to the height of $130 \mathrm{~cm}$ above the floor of the semi-anechoic room. The probe was placed inside the enclosure. Its height above the floor was maintained. The transmitting antenna was positioned to the same height, i.e. $130 \mathrm{~cm}$. The transmitting antenna was oriented in horizontal polarization based on our experience that horizontal polarization of the transmitting antenna placed in this semi-anechoic room leads to lower reflection occurrence compared to the vertical polarization [22]. The experiment configuration inside the semi-anechoic room is depicted in Fig.5. and Fig.6.

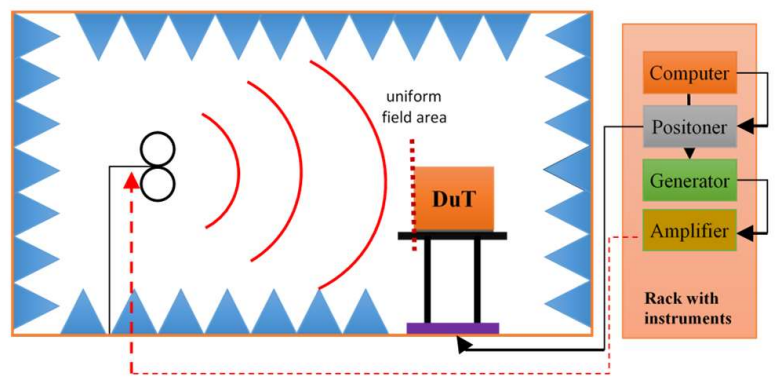

Fig.7. Typical setup according to the standard EN 61000-4-3 [23].

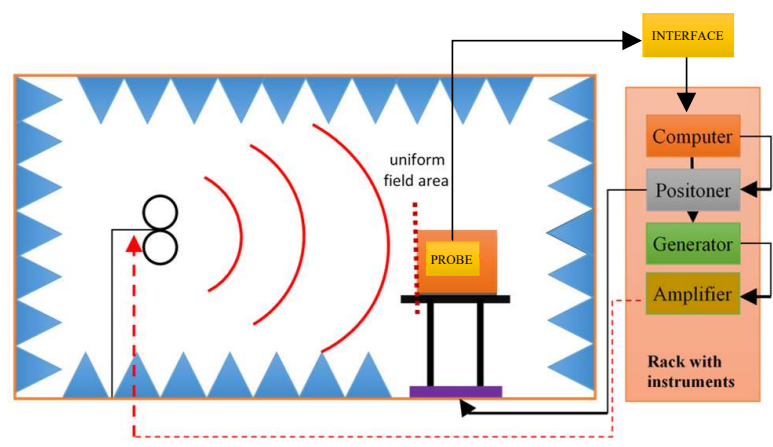

Fig.8. Extended setup for the purposes of the hereby described experiment.
As mentioned above, the experiment was conducted under the laboratory configuration in accordance with the standard EN 61000-4-3. The typical configuration for susceptibility to electrical field testing is depicted in Fig.7. The transmitting antenna and the measured device are placed inside the anechoic room. The generator, amplifier and monitoring systems are controlled by one computer, using EMC 32 software. For the purposes of the hereby described experiment, the configuration of the laboratory site was extended according to Fig.8. The limits of the configuration were given by the configuration possibilities of the whole laboratory system.

\section{EXPERIMENT STEPS AND DATA POSTPROCESSING}

The experiment was conducted in two main steps. In the first step, the configuration setup according to Fig.5. was utilized in order to measure the intensity of the electric field at the point where the probe without the shielding enclosure was placed. The antenna was driven by a sinusoidal signal the power of which was constant, $P_{l}=35 \mathrm{~W}$. The frequency of the transmitted signal was changed from $200 \mathrm{MHz}$ up to $3 \mathrm{GHz}$ with the steps of $1 \%$. In total, 276 transmitting frequencies were applied. The field intensity measured by the E-field probe was between 15 and $30 \mathrm{~V} / \mathrm{m}$ according to the frequency of the transmitted signal. For each of the transmitted frequencies, the field intensity measured by the probe was recorded by the controlling system.

In the second step, the shielding enclosure was employed. The E-field probe was placed inside the enclosure to the same height as in the first step (130 cm above the floor). This setup is depicted in Fig.6. During this step, the following issue was observed: the E-field probe is primarily used as a feedback probe when performing EMC susceptibility tests according to EN 61000-4-3 or system calibration. The controlling software expects the levels in tens of Volts per meter. Once the probe was placed inside the enclosure, the measured signal level, compared to the output power of the amplifiers, was low enough to enable system error shutdown. Therefore, in the second step, different setting of the controlling software was used. The system was set to generate the field intensity at least $0.1 \mathrm{~V} / \mathrm{m}$ by setting the output power of the amplifiers for each of the transmitting frequencies individually. The maximum output power of the amplifier AR $150 \mathrm{~W} 1000 \mathrm{~A}$ is $150 \mathrm{~W}$ up to $1 \mathrm{GHz}$ [24] and the maximum output power of the amplifier AR $80 \mathrm{~S} 1 \mathrm{G} 4$ is $80 \mathrm{~W}$ up to $4 \mathrm{GHz}$ [25]. During this measurement, a data record was obtained, consisting of the following information:

1. Forward output power of the amplifiers for each of the transmitting frequency. Almost in all cases, the output power was set to the maximum level. It must be noted that the laboratory system enables not only measurement of the gross output power of the amplifiers, but it also measures the transmitted and the reflected power. For the purposes of our calculations, the information on transmitted power was utilized.

2. Difference between the required level of $0.1 \mathrm{~V} / \mathrm{m}$ and the level of the field inside the enclosure measured by the E-probe. 
According to the setting described above, the system error occurrence was depreciated, but different datasets for step 1 and step 2 were obtained. To ensure comparability of the data, further processing was necessary in order to normalize the measured values. Let us assume the number of frequencies at which the measurement was conducted is $k=276$. Provided the electrical field intensities $E_{l, 1} \ldots E_{l, k}$ measured for each of the frequencies in the $1^{\text {st }}$ step were obtained with a constant transmitting power $P_{I}$ and the field intensities $E_{2,1} \ldots E_{2, k}$ were obtained with a fluctuating transmitting power $P_{2, n}$, where $n \in\langle 1, k\rangle$, the following equation may be used to calculate the shielding effectiveness in decibels for each of the frequencies:

$$
S E_{d B n}=20 \log _{10}\left(\frac{E_{1, n}}{E_{2, n}} \cdot \sqrt{\frac{P_{2, n}}{P_{1}}}\right), n \in\langle 1, k\rangle
$$

Within the frequency range from $200 \mathrm{MHz}$ up to $3 \mathrm{GHz}$, the average shielding effectiveness $S E_{a v g}$ was calculated as follows:

$$
S E_{\text {avg }}=20 \log _{10}\left(\frac{1}{k} \cdot \sum_{n=1}^{k}\left(\frac{E_{1, n}}{E_{2, n}} \cdot \sqrt{\frac{P_{2, n}}{P_{1}}}\right)\right)
$$

According to (10), the calculated average shielding effectiveness within the range from $200 \mathrm{MHz}$ up to $3 \mathrm{GHz}$ was as high as $53.1 \mathrm{~dB}$.

\section{RESULTS \& DISCUSSION}

Considering the measurement of EM field intensities, great $B$ type uncertainties are usually expected and tolerated. The field intensity may vary in time and space. Moreover, the shape and parameters of measurement antennas and rooms introduce a large error that is not easy to describe exactly, at least because of their mutual interactions. The parameters of the measured field are frequency dependent as well as various interactions between the devices, resulting in quite unpredictable frequency dependence of the measured values. Various approximating techniques are still subject to research, as evident for example from [7].

Another example is given in [26] where highly appreciated scientists in the field of shielding effectiveness introduce results of their measurements. The reported values vary according to the frequency up to $\pm 10 \mathrm{~dB}$, which is given by a huge number of factors.

In practice, the EMC laboratories that certify electronic devices must fulfil the requirements enlisted in a family of EN 55016 standards. The part 4 of EN 55016 is called "Uncertainties, statistics and limit modelling" and is divided into 4 subdocuments. In the document EN 55016-4-2, called "Measurement instrumentation uncertainty", there is a large list of expected B type uncertainties that occur in the EMC laboratories. These uncertainties are defined for each of the measurements separately. For example, for antenna measurements in far field in the frequency range from $30 \mathrm{MHz}$ up to $1 \mathrm{GHz}$, using a biconical antenna, there are 19 factors defined in total. The total expanded B type uncertainty is $5.06 \mathrm{~dB}$ in this case, provided the measurement distance is $3 \mathrm{~m}$. Among the factors that cause measurement uncertainty, for example, the following ones are listed: antenna factor uncertainty $( \pm 2 \mathrm{~dB})$, imperfection of the measuring site $( \pm 4 \mathrm{~dB})$, uncertainty caused by antenna distance deviation $( \pm 0.3 \mathrm{~dB})$, and many others. However, the antenna factor uncertainty and the imperfection of the measuring site that covers residual reflections inside the semi-anechoic room are the greatest ones.

Although the method of utilization of the E-field probe, described in this paper, is not included in EN 55016, we are convinced that the measurement uncertainty is similar to the typical values described in EN 55016, as we employed the same laboratory equipment. Due to low levels of the measured signal when the shielding cover was applied, it can be expected that the background noise effect would increase in importance. On the other hand, the uncertainty caused by imperfection of the measuring site would not occur, as the hereby described method is based on comparative measurement, which exclude this kind of uncertainties in principle.

Based on the discussion above, we are convinced that the measurement uncertainty that occurred within this experiment can be expected to be as high as typical uncertainties described in EN 55016, which vary from approximately 3.5 to approximately $6.3 \mathrm{~dB}$.

According to the data depicted in Fig.4., the average shielding effectiveness measured in the frequency range from $200 \mathrm{MHz}$ up to $3 \mathrm{GHz}$ was $68.9 \mathrm{~dB}$ for the metal plate and $46.5 \mathrm{~dB}$ for the Technical fibre TT-0,630-0,25-1.4401-1000. Therefore, the measured average shielding effectiveness of the real enclosure as high as $53.1 \mathrm{~dB}$ can be, according to our opinion, considered as realistic, especially when the abovementioned facts are considered.

\section{CONCLUSIONS}

In this paper, a description of an experiment on the measurement of the shielding effectiveness of an enclosure for a security camera is described. The main purpose of the enclosure is to ensure the susceptibility of a security camera to external electromagnetic interferences.

In accordance with the possibilities of our laboratory equipped with the semi-anechoic room Frankonia SAC3+ and a testing system for processing electromagnetic susceptibility tests according to the standard EN 61000-4-3, the indirect measurement employing the calibration E-field probe was used. The final results were calculated from the error calibration data, when the testing system with E-field probe blinded by the shielding enclosure tried to recalibrate the required field levels inside the semi-anechoic room.

The shielding enclosure was made of two different materials: a standard stainless metal plate and a technical fibre allowing the camera to see the picture outside the enclosure. Details on the construction are described in [6]. Prior to this experiment, the shielding effectiveness of the construction materials was measured, utilizing the method with flanged coaxial cables [19]. Based on these results, the estimated shielding effectiveness was at least $50 \mathrm{~dB}$. 
After making the assumptions, two measurements were performed in order to verify the expected shielding effectiveness level as well as to verify the applicability of the construction proposed in [6]. One measurement was processed with the probe placed in a free space of the additionally damped semi-anechoic room and the second one with the probe placed inside the constructed enclosure. Within the frequency range from $200 \mathrm{MHz}$ up to $3 \mathrm{GHz}$, the average shielding effectiveness level was $53.1 \mathrm{~dB}$, which is in accordance with the expected levels.

efficiency enhancement of the PANI-CSA Two main interesting conclusions may be given based on the results of the hereby described experiment:

1. The shielding enclosure the design and construction of which are described in [6] is suitable for shielding the security cameras from external electromagnetic field interferences. By means of the shielding enclosure, the security camera susceptibility to an external electromagnetic field can be considered as highly increased.

2. The results of the hereby described experiment indicate the possibility of indirect shielding effectiveness measurement through standard EMC laboratory equipment. Despite quite high B type uncertainties the typical values of which are defined in EN 55016, the results may be, in comparison to other methods mentioned in this paper, according to our opinion, considered at least as indicative.

Our future work is aimed at verification of the measured results by means of CST Microwave Studio software. It is expected that the simulations performed in this software could lead to an increase in accuracy of the hereby described method.

\section{ACKNOWLEDGMENT}

This paper is supported by the Ministry of Education, Youth and Sports of the Czech Republic within the National Sustainability Programme Project No. LO1303 (MSMT7778/2014).

\section{REFERENCES}

[1] Paul, C.R. (2006). Introduction to Electromagnetic Compatibility (2nd ed). Wiley-Interscience, ISBN 0471-75500-1.

[2] Maxwell, J. (2010). A Treatise on Electricity and Magnetism: Volume 2. Cambridge University Press, ISBN 978-0511709340.

[3] Das, A., Kothari, V.K., Kothari, A., Kumar, A. (2009). Effect of various parameters on electromagnetic shielding effectiveness of textile fabrics. In Indian Journal of Fibre \& Textile Research, 34 (2), 144-148.

[4] Raj, C.D., Rao, G.S., Jayasree, P.V.Y., Srinu, B., Lakshman, P. (2010). Estimation of reflectivity and shielding effectiveness of three layered laminate electromagnetic shield at X-band. In Progress in Electromagnetics Research B, 20, 205-223.

[5] Sasikumar, S.P., Libimol, V.A., George, D.M., Lindo, A.O., Pushkaran, N.K., John, H., Aanandan, C.K. (2017). Electromagnetic interference shielding films at broad band frequencies. In Progress in Electromagnetics Research M, 57, 163-174.
[6] Skočík, P. (2020). Resistant Camera System Designed for Operation in Anechoic Shielded Chambers. Unpublished doctoral dissertation, Tomas Bata University in Zlín, Faculty of Applied Informatics, Czech Republic.

[7] Dawson, J.F., Marvin A.C., Robinson M.P., Flintoft I.D. (2018). On the meaning of enclosure shielding effectiveness. In 2018 International Symposium on Electromagnetic Compatibility (EMC EUROPE). IEEE, 746-751.

[8] Pospíšilík, M., Soldán, J., Křesálek, V., Adámek, M., Silva, R.M.S. (2015). Measurement of a shielding effectiveness of composite materials according to MILSTD 285 and IEEE Std 299-2006. In XXI IMEKO World Congress "Measurement in Research and Industry". IMEKO, ISBN 978-80-01-05793-3.

[9] Kovář, S., Valouch, J., Adámek, M. (2016). Electromagnetic susceptibility of IP camera. Przeglad Elektrotechniczny, 92 (5), 204-208.

[10] Pospíšilík, M., Ríha, T., Adámek, M., Silva, R.M.S. (2015). DSLR camera immunity to electromagnetic fields - experiment description. WSEAS Transactions on Circuits and Systems, 14 (1), 494-504.

[11] IEEE. (2007). IEEE 299-2006 - IEEE Standard Method for Measuring the Effectiveness of Electromagnetic Shielding Enclosures.

[12] Schelkunoff, S.A. (1943). Electromagnetic Waves. D.Van Nostrand Company. ISBN 978-1124119366.

[13] Svačina, J. (2001). Electromagnetic Compatibility: Lectures. Brno, Czech Republic: Brno University of Technology, ISBN 80-214-1573-8.

[14] Pospíšilík, M., Soldán, J., Adámek, M. (2015). Influence of the properties of a real semi anechoic chamber on an internal electromagnetic field distribution. In WSEAS Transactions on Systems, 14, 175-186.

[15] Pospíšilík, M., Silva, R., Adámek, M. (2016). Maple algorithm for damping quality of anechoic chambers evaluation. In International Journal of Mathematics and Computers in Simulation, 10, 161-170.

[16] Witkovský, V., Frollo, I. (2020). Measurement science is the science of sciences - there is no science without measurement. Measurement Science Review, 20 (1), $1-5$.

[17] Dawson, J.F., Konefal, T. Robinson, M.P., Marvin, A.C., Porter, S.J., Chirwa, L.C. (2005). Field statistics in an enclosure with an aperture - effect of Q-factor and number of modes. In 2005 International Symposium on Electromagnetic Compatibility (EMC 2005). IEEE, 141-146.

[18] Sewell, P., Turner, J.D., Robinson, M.P., Benson, T.M., Christopoulos, C., Dawson, J.F., Ganley, M.D., Marvin, A.C., Porter, S.J. (1998). Comparison of analytic, numerical and approximate models for shielding effectiveness with measurement. In IEE Proceedings Science, Measurement and Technology, 145 (2), 61-66.

[19] Dř́novský, J., Kejík, Z. (2009). Electromagnetic shielding efficiency measurement of composite materials. Measurement Science Review, 9 (4), 109112 . 
[20] ASTM. (2018). Standard Test Method for Measuring the Electromagnetic Shielding Effectiveness of Planar Materials. ASTM D4935 - 18.

[21] ETS Lindgren. (2019). ETS-Lindgren HI-6105 Electric Field Probe - Data Sheets. http://www.etslindgren.com/datasheet/probes-monitors/emc-probes/ electric-field-probes/9003/900304.

[22] Pospíšilík, M., Adámek, M., Neumann, P. (2018). Influence of the antenna's height to the standing waves ratio when performing the electromagnetic susceptibility tests in anechoic chambers. In Applied Physics, System Science and Computers: Proceedings of the 1st International Conference on Applied Physics, System Science and Computers (APSAC2016), September 28-30, Dubrovnik, Croatia. Springer, LNEE 428, 161-167.
[23] Pospíšilík, M. (2019). Introduction to Electromagnetic Compatibility for Electronic Engineers: ... and not only for them. Zlin, Czech Republic: Tomas Bata University in Zlin, ISBN 978-80-7454-876-5.

[24] AR RF/Microwave Instrumentation. AR 150W1000A RF Power Amplifiers - Data Sheets. https://www.arworld.us/post/150W1000A.pdf.

[25] AR RF/Microwave Instrumentation. AR 80S1G4 Microwave Solid State Amplifiers - Data Sheets. https:/www.arworld.us/post/80S1G4.pdf.

[26] Leferink, F.B.J., Bergsma, H., Van Etten, W.C. (2006). Shielding effectiveness measurements using a reverberation chamber. In 2006 17th International Zurich Symposium on Electromagnetic Compatibility. IEEE, 505-508.

Received August 08, 2020

Accepted February 02, 2021 